\title{
SEGURIDAD Y SALUD: UNA NUEVA PERSPECTIVA DE LOS SISTEMAS DE GESTIÓN DE SEGURIDAD Y SALUD CON MOTIVO DE LA APARICIÓN DE RIESGOS EMERGENTES
}

\author{
Karla Canova Talledo* \\ Universidad de Lima, Lima, Perú \\ Recibido: 13/2/2021 Aceptado: 20/2/2021 \\ doi https://doi.org/10.26439/iusetpraxis2021.n053.4973
}

\begin{abstract}
RESUMEN. Con motivo del uso masivo de las tecnologías, la robótica, la intervención de la inteligencia artificial, la automatización, la aparición de nuevos riesgos biológicos, entre otros, las relaciones laborales vienen evolucionando de una manera muy particular, que obliga a que los sistemas de gestión de seguridad y salud en el trabajo vayan de la mano en este proceso. Por ello, el presente artículo desarrolla el marco de los riesgos emergentes y la necesidad de que nuestros sistemas de gestión se articulen y evolucionen desde todas sus perspectivas.
\end{abstract}

PALABRAS CLAVE: sistemas de gestión de seguridad y salud / riesgos emergentes / inteligencia artificial / riesgos biológicos / trabajo remoto / teletrabajo

\section{SAFETY AND HEALTH: A NEW PERSPECTIVE OF THE SYSTEMS OF SECURITY MANAGEMENT AND HEALTH ON THE OCCASION OF APPARITION OF EMERGING RISKS}

ABSTRACT. Due to the massive use of technologies, robotics, the intervention of artificial intelligence, automation, the appearance of new biological risks, among others, labor relations have been evolving in a very particular way, which requires that the Occupational Health and Safety Management go hand in hand in this process. This article develops the framework of emerging risks and the need for our management systems to be articulated and evolve from all their perspectives.

KEYWORDS: health and safety management systems / emerging risks / artificial intelligence / biological risks / remote work / teleworking

\footnotetext{
* Abogada por la Universidad de Lima, Perú. Máster en Seguridad y Salud Ocupacional por la Universidad de Turín, Italia. Egresada de la Maestría de Relaciones Laborales por la Pontificia Universidad Católica del Perú. Especialista en Derecho del Trabajo por la Universidad de Sevilla, España. Se desempeña como docente en la Universidad de Lima y en la Escuela de Posgrado de la UPC. Actual directora de Seguridad y Salud en el Trabajo del Ministerio de Trabajo y Promoción del Empleo, y directora ejecutiva de la Secretaría Administrativa Permanente de la Asociación Latinoamericana de Salud Ocupacional (ALSO).
} 


\section{INTRODUCCIÓN}

En el actual contexto, el mundo atraviesa una serie de cambios con motivo del riesgo biológico por el virus SARS-CoV-2, que causa la enfermedad del COVID-19, por lo que nos encontramos en el marco de una emergencia sanitaria sin precedentes. Esta pandemia ha llevado a que los sistemas de gestión de seguridad y salud en el trabajo sean de especial atención para el Estado, como aliados estratégicos para mitigar el impacto de este riesgo biológico.

Entre las medidas adoptadas, se han generado nuevas figuras laborales como el trabajo remoto, y otras han cobrado mayor interés, como el teletrabajo, que fue regulado desde el año 2013. Si bien es cierto que estas figuras laborales se impusieron como alternativas al riesgo biológico, también han provocado riesgos emergentes. Por ello, es importante exponerlos a fin de empezar a mejorar el diseño de nuestros sistemas de gestión en respeto al derecho fundamental a la seguridad y salud en el trabajo.

\section{EL SISTEMA DE SEGURIDAD Y SALUD EN EL MARCO NORMATIVO PERUANO}

Según lo establece la Organización Internacional del Trabajo (OIT), la gestión de la seguridad y la salud forma parte de la gestión de una empresa. Las empresas deben hacer una evaluación para conocer cuáles son los peligros y los riesgos en sus lugares de trabajo, y adoptar medidas para controlarlos con eficacia, asegurando que dichos peligros y riesgos no causen daños a los trabajadores (OIT, s. f.).

En el Estado peruano contamos con un marco normativo específico dispuesto por la Ley 29783, Ley de Seguridad y Salud en el Trabajo, y su reglamento Decreto Supremo 005-2012-TR, así como disposiciones complementarias que establecen que el empleador se obligue a implementar un sistema de gestión de seguridad y salud en el trabajo atendiendo al "deber de prevención de riesgos laborales", lo que genera derechos y obligaciones en las partes intervinientes.

Asimismo, el rol del Estado en el marco de los sistemas de gestión de seguridad y salud resulta fundamental considerando su función orientadora y fiscalizadora, que contribuye al respeto de este derecho fundamental que tiene todo trabajador por la sola existencia de una relación laboral. De la misma manera, es el derecho de todo joven bajo modalidad formativa o locador de servicios que se encuentre realizando alguna actividad en un centro de trabajo o por encargo de un empleador.

En esta línea, la Ley 29783, Ley de Seguridad y Salud en el Trabajo, dispone en su artículo 17 que "el empleador debe adoptar un enfoque de sistema de gestión en el área de seguridad y salud en el trabajo, de conformidad con los instrumentos y directrices internacionales y la legislación vigente". Asimismo, dispone en su artículo 18, como principios rectores del sistema de gestión de seguridad y salud en el trabajo, los siguientes: 
a) Asegurar un compromiso visible del empleador con la salud y seguridad de los trabajadores.

b) Lograr coherencia entre lo que se planifica y lo que se realiza.

c) Propender al mejoramiento continuo, a través de una metodología que lo garantice.

d) Mejorar la autoestima y fomentar el trabajo en equipo a fin de incentivar la cooperación de los trabajadores.

e) Fomentar la cultura de la prevención de los riesgos laborales para que toda la organización interiorice los conceptos de prevención y proactividad, promoviendo comportamientos seguros.

f) Crear oportunidades para alentar una empatía del empleador hacia los trabajadores y viceversa.

g) Asegurar la existencia de medios de retroalimentación desde los trabajadores al empleador en seguridad y salud en el trabajo.

h) Disponer de mecanismos de reconocimiento al personal proactivo interesado en el mejoramiento continuo de la seguridad y salud laboral.

i) Evaluar los principales riesgos que puedan ocasionar los mayores perjuicios a la salud y seguridad de los trabajadores, al empleador y otros.

j) Fomentar y respetar la participación de las organizaciones sindicales -o, en defecto de estas, la de los representantes de los trabajadores- en las decisiones sobre la seguridad y salud en el trabajo.

De lo expuesto, resulta importante resaltar la obligación que tienen los actores (empleadores y trabajadores) de contribuir a fin de que el sistema de seguridad y salud sea eficiente y cumpla con el rol de la prevención. Asimismo, se dispone un mejoramiento continuo, que implica la evaluación y vigilancia permanente de los riesgos laborales que podrían afectar la salud de los trabajadores. Consecuentemente, este sistema de gestión resulta ser dinámico y va de la mano con los cambios que puedan surgir de las actividades o puestos de trabajo y los cambios que puedan surgir en el entorno de trabajo.

Siendo esto así, el empleador se obliga, en el marco del deber de prevención, a considerar y evaluar los riesgos emergentes que actualmente se encuentran en pleno auge por la aparición de un nuevo riesgo biológico. Por ello, los sistemas productivos han buscado alternativas y han migrado de manera exponencial al uso masivo de la tecnología, la robótica, la inteligencia artificial y la nanotecnología, entre otros recursos que los avances en las ciencias nos traen.

Claro ejemplo de esta migración es la creación del Plan de Vigilancia y Control del COVID-19, regulado por la Resolución Ministerial 448-2020-MINSA, que dispone medidas específicas en el centro de trabajo con motivo del riesgo biológico y que conduce a que los empleadores se obliguen a potenciar su sistema de gestión de seguridad y salud. 
Para ello, deberán observar la conformación de los servicios de seguridad y salud, la actuación del supervisor o comité, reforzar las capacitaciones a los trabajadores utilizando plataformas digitales, entre otros aspectos.

\section{RIESGOS EMERGENTES}

Según la Agencia Europea para la Seguridad y Salud (2014), el trabajo y los lugares de trabajo están sometidos a cambios continuos por la introducción de nuevas tecnologías, sustancias y procesos de trabajo; por cambios en la estructura de la población activa y del mercado laboral; y por formas nuevas de empleo y organización del trabajo. Todo lo anterior puede dar lugar a que aparezcan nuevos riesgos y dificultades para la seguridad y la salud en el trabajo (SST), que es necesario prever y abordar para garantizar la SST en el futuro.

Ahora bien, es importante adoptar una definición coherente de este supuesto. Por ello, según Mairal (2013), se entiende por riesgo emergente cualquier riesgo nuevo que va en aumento.

Entendiendo por "nuevo":

- el riesgo no existía anteriormente y está causado por nuevos procesos, tecnologías o tipos de lugar de trabajo, o por cambios sociales u organizativos; o que

- se trata de un problema persistente que pasa a considerarse como un riesgo debido a un cambio en las percepciones sociales o públicas; o que

- un nuevo conocimiento científico da lugar a que una cuestión no novedosa se identifique como riesgo.

Y entendiendo por "emergente":

- aumenta el número de factores de peligro que dan lugar al mismo,

- la exposición al factor de peligro que da lugar al riesgo aumenta (nivel de exposición y número de personas expuestas), o

- el efecto del factor de peligro sobre la salud de los trabajadores empeora (gravedad de los efectos sobre la salud y número de personas afectadas). (Mairal, 2013, párrs. 4-5)

Conocer y evaluar con antelación estos riesgos resulta fundamental para el desarrollo de la seguridad y salud en el país a fin de que se adopten las medidas adecuadas que permitan la adaptación de los sistemas, de la normativa y de cualquier política pública que tenga que mejorarse para poder cumplir con el rol de la prevención de riesgos laborales dispuesta en toda política nacional de seguridad y salud en el trabajo. 
A manera de ejemplo, la EU-OSHA encargó un Estudio prospectivo sobre los riesgos nuevos y emergentes asociados a las nuevas tecnologías en 2020 (Agencia Europea para la Seguridad y Salud en el Trabajo, 2014), en el cual se empleó el método de creación de escenarios para analizar la evolución de diversas tecnologías relacionadas con los empleos verdes y los riesgos para la seguridad y salud en el trabajo asociados a las mismas, en tres situaciones futuras posibles para Europa. Todo esto conduce a que podamos identificar y ajustar nuestras políticas a la realidad en que nos encontramos, y a que los sistemas de gestión de seguridad y salud evolucionen junto con las relaciones laborales, que en este contexto han tenido que migrar de manera exponencial (Sanguineti, 2020), utilizando diversas formas que analizaremos más detenidamente en el presente artículo.

\section{NUEVAS RELACIONES LABORALES Y LOS RIESGOS EMERGENTES}

Con la detección del primer caso de COVID-19 en el Perú el 6 de marzo del 2020, se buscaron nuevas fórmulas legales en materia laboral para proteger a los trabajadores del sector privado y a los servidores públicos, analizando la implementación del trabajo a domicilio o el teletrabajo (figuras ya contempladas en la normativa nacional). Sin embargo, dichas modalidades laborales fueron pensadas para utilizarse en circunstancias ajenas al riesgo biológico que representa el virus del SARS-CoV-2, por lo que su propia regulación fue el primer problema para ponerlas en práctica de forma masiva durante la declaratoria de emergencia sanitaria en nuestro país, aprobada por el Decreto Supremo 008-2020-SA.

Por un lado, la principal dificultad de aplicar la modalidad de trabajo a domicilio residía en la imposibilidad de que el empleador supervisara de forma directa e inmediata al trabajador; y con la aplicación del teletrabajo - al tener el teletrabajador los mismos derechos y obligaciones que los establecidos para los trabajadores del régimen laboral de la actividad privada-, el empleador debía implementar las medidas de protección en materia de SST en el lugar donde el teletrabajador presta el servicio (domicilio o lugar de aislamiento), por lo que estos lugares tenían que reunir las condiciones óptimas para teletrabajar; por tanto, el empleador estaría obligado a supervisar cada domicilio de sus trabajadores a fin de implementar las medidas correspondientes en materia de SST, todo ello en plena expansión del COVID-19. Por estas razones, en nuestro país se implementa una figura laboral más flexible a la cual se le denominó trabajo remoto, como otra de las formas de trabajo a distancia. Veamos los alcances de estas figuras en el marco de la seguridad y salud como derecho fundamental. 
Tabla 1

Comparación entre las figuras de teletrabajo y trabajo remoto

\begin{tabular}{ll}
\hline Teletrabajo & Trabajo remoto \\
\hline Se consolida con la voluntad de las & Es facultad de los empleadores del sector público y privado \\
partes. & modificar el lugar de la prestación de servicios de sus \\
& trabajadores. Esta decisión debe ser comunicada al trabajador \\
& mediante soporte físico o digital.
\end{tabular}

Se trata del desempeño subordinado de labores sin la presencia física del trabajador, denominado teletrabajador, en la empresa con la que mantiene vínculo laboral, a través de medios informáticos, de telecomunicaciones y análogos, mediante los cuales se ejerce a su vez el control y la supervisión de las labores.

\begin{abstract}
Se trata de la prestación de servicios subordinada con la presencia física del trabajador en su domicilio o lugar de aislamiento domiciliario, utilizando cualquier medio o mecanismo que posibilite realizar las labores fuera del centro de trabajo, siempre que la naturaleza de las labores lo permita. Los equipos y medios informáticos, de telecomunicaciones y análogos (internet, telefonía u otros), así como de cualquier otra naturaleza que resulten necesarios para la prestación de servicios, pueden ser proporcionados por el empleador o por el trabajador.
\end{abstract}

Elaboración propia

De lo expuesto hasta aquí, tanto en el teletrabajo como en el trabajo remoto, figuras mayormente utilizadas en este contexto, no se advierten disposiciones específicas en materia de seguridad y salud en el trabajo, con lo cual no puede inobservase lo dispuesto en la Ley 29783, disposición normativa transversal que debe respetarse en cualquier modalidad de trabajo a distancia. Siendo esto así, las principales interrogantes que surgen son estas: ¿cómo opera el sistema de gestión de seguridad y salud en el trabajo en protección de estos trabajadores en figuras de trabajo a distancia ante la aparición de riesgos emergentes? ¿Puede configurarse un accidente de trabajo en el trabajo a distancia? ¿Existe responsabilidad del empleador?

Como ya se expuso anteriormente, el empleador es responsable del sistema de gestión de seguridad y salud en el trabajo; por ello, también se hace responsable de los trabajadores que en este contexto de pandemia se encuentren ante alguna de las formas de trabajo a distancia. En esta línea, es indispensable que el empleador evalúe los riesgos y peligros a los que se encuentre expuesto el trabajador en el espacio que haya sido asignado para la prestación de las labores. Para ello, podría coordinar una visita presencial con el trabajador o, mejor aún, hacer uso de los recursos tecnológicos para la detección de peligros y riesgos del lugar de trabajo. De esta manera, podrá emitir las recomendaciones correspondientes para evitar cualquier afectación a la salud del trabajador. Es importante, además, resaltar la obligación de parte del trabajador de cumplir con las disposiciones que emita el empleador. 
Por otro lado, en atención a la interrogante sobre la configuración de un accidente de trabajo durante la prestación de las labores en el marco de una de las modalidades de trabajo a distancia, en efecto, puede configurarse y será el empleador quien deberá cubrir cualquier atención que requiera el trabajador hasta su recuperación e inserción al trabajo, como bien lo disponen los principios de responsabilidad y atención integral de la salud dispuestos en el "Título preliminar" de la Ley 29783. Lo afirmado hasta aquí coincide con el derecho comparado; por ejemplo, en España opera la "presunción de laboralidad del accidente de trabajo"; en Chile, también tenemos un antecedente que se recoge de la reciente Ley 21220, dispositivo que modifica el Código del Trabajo en materia de trabajo a distancia y mantiene una "suerte" de presunción de laboralidad del accidente de trabajo, toda vez que, en un primer momento, el trabajador tendrá cobertura integral de la atención de parte de la aseguradora sin mayores demoras y, posteriormente, se evaluará la naturaleza del accidente.

Sanguineti (2020) señala cuatro situaciones o fenómenos negativos típicos del estado actual de las cosas respecto del teletrabajo y trabajo remoto, que deberían evitarse para garantizar la continuidad de esta figura en el marco del irrestricto respeto por los derechos de los trabajadores:

1. El trabajo remoto impuesto, sin dotación de medios ni adaptación del lugar de trabajo y con asunción de sus costes por el trabajador.

2. La invasión del domicilio y la vida privada y familiar del trabajador, así como su intimidad y privacidad, a través del uso de medios tecnológicos.

3. La desaparición de las fronteras entre el trabajo y la vida personal, social y familiar.

4. El aislamiento y la falta de relaciones personales y sociales directas.

Todo lo anterior opera en el marco de la responsabilidad del empleador frente al sistema de gestión e, igualmente, frente a los riesgos que se generan. Entre estos encontramos:

1. Trastornos psicosociales derivados de la sobrecarga laboral, aislamiento para el trabajo, doble rol (especialmente en el caso de las mujeres que trabajan), la sobrevigilancia, entre otros.

2. Trastornos musculoesqueléticos derivados de las condiciones inadecuadas para el trabajo o de las malas posturas provocadas por equipos insuficientes para proteger la salud de los trabajadores.

Estos problemas se han agudizado en el uso de estas nuevas figuras laborales. Para mejorarlos, el empleador debe gestionar los riesgos para mitigar el impacto en la salud de los trabajadores. Esto implica evaluar el diseño de su sistema de gestión de seguridad y salud en el trabajo, el cual no solo debe limitarse a la ocurrencia de accidentes de 
trabajo o enfermedades profesionales, sino que debe ir más allá y comprender aspectos relacionados con riesgos emergentes en el marco del principio de prevención.

\section{REFLEXIONES FINALES}

1. Con motivo de la emergencia sanitaria, se destaca la necesidad de dinamizar y actualizar constantemente los sistemas de gestión de seguridad y salud en el trabajo, debido a la aparición de nuevos riesgos laborales.

2. El uso de tecnología, digitalización, introducción de comunicación digitalizada, inteligencia artificial, robótica y nanotecnología conduce a que los sistemas de gestión de seguridad y salud también se reinventen a fin de poder enfrentar los riesgos derivados de estos cambios.

3. El respeto por el derecho fundamental de la seguridad y salud se extiende a cualquier modalidad que haya sido creada en el marco del trabajo a distancia (teletrabajo y trabajo remoto), y deberá evolucionar atendiendo a la aparición de riesgos emergentes. A la vez, las disposiciones legales, así como las políticas públicas, también deberán adaptarse a fin de cumplir con el principal objetivo que es la prevención de riesgos laborales.

4. Tanto en el teletrabajo como en el trabajo remoto o en cualquier modalidad de trabajo a distancia, no se puede perder de vista el derecho a la vigilancia de la salud de los trabajadores y al reclamo por cualquier daño que se origine a su integridad física o psicológica durante la ejecución de sus labores.

5. El empleador no solo debe limitarse al análisis de peligros y riesgos del puesto de trabajo, sino que, además, alcanza al entorno de trabajo, más aún cuando el contexto actual nos lleva a tener a la "seguridad y salud en casa".

6. Resulta posible que se configure un accidente de trabajo o enfermedad profesional ante cualquiera de los escenarios de trabajo a distancia, para lo cual rige el principio de laboralidad del accidente, analizándose cada caso en concreto respecto de la causalidad.

7. Los sistemas de gestión de seguridad y salud no pueden perder de vista el enfoque de género dispuesto en la Ley 29783 a fin de disminuir las brechas y buscar la integración eficiente de la mujer en el trabajo, observando los riesgos emergentes en el marco de las nuevas figuras laborales. 


\section{REFERENCIAS}

Agencia Europea para la Seguridad y la Salud en el Trabajo. (2014). Estudio prospectivo sobre los riesgos nuevos y emergentes asociados a las nuevas tecnologías en 2020. Taller dirigido a los centros de referencia de la UE. https://osha.europa. eu/es/publications/foresight-new-and-emerging-risks-associated-newtechnologies-2020-workshop-eu-focal

Mairal, D. (17 de septiembre del 2013). ¿Qué son los riesgos emergentes? PrevenBlog. https://prevencontrol.com/prevenblog/sabes-que-son-los-riesgosemergentes/

Oficina Internacional de Trabajo; y Ministerio de Asuntos Sociales. (1998). Enciclopedia de Seguridad y Salud en el Trabajo. Organización Internacional del Trabajo; Ministerio de Trabajo y Asuntos Sociales de España. https://www.insst.es/ documents/94886/161958/Cap\%C3\%ADtulo+16.+Servicios+de+salud+en+el+tr abajo

Organización Internacional del Trabajo. (2019). Seguridad y salud en el centro del futuro del trabajo. Aprovechar 100 años de experiencia. https://www.ilo.org/wcmsp5/ groups/public/---dgreports/---dcomm/documents/publication/wcms_686762. pdf

Organización Internacional del Trabajo. (s. f.). ¿Cómo gestionar la seguridad y salud en el trabajo? Recuperado el 12 de noviembre del 2021 de https://www.ilo.org/global/ topics/labour-administration-inspection/resources-library/publications/guidefor-labour-inspectors/how-can-osh-be-managed/lang--es/index.htm

Organización Mundial de la Salud. (2010). Ambientes de trabajo saludables: un modelo para la acción para empleadores, trabajadores, autoridades normativas y profesionales. https://apps.who.int/iris/bitstream/handle/10665/44317/9789243599311_spa. pdf;jsessionid=61B103CA630837801E95641DDE6CDC32? sequence $=1$

Sanguineti, W. (2002). Teletrabajo y globalización: en busca de respuestas al desafío de la transnacionalización del empleo [Informe]. https://wilfredosanguineti.files. wordpress.com/2009/08/teletrabajo-y-globalizacion-final-informe.pdf

Sanguineti, W. (2020). ¿La hora del teletrabajo? Trabajo y Derecho, 66. 
